\title{
INVARIANTS OF THE ANTI-AUTOMORPHISMS OF A GROUP
}

\section{J. RICHARD BÜCHI AND JESSE B. WRIGHT}

1. Introduction. The background for this paper is provided by Klein's work presented in the Erlangerprogram [1], and more recent developments of these ideas as well as their application outside the field of geometry $[2 ; 3 ; 4 ; 5 ; 6]$.

Klein deals with the Euclidean space $\bar{A}$ as a fundamental structure. Its group of automorphisms $G_{0}(\bar{A})$ consists of the similitudes. Let $G(\bar{A})$ denote any group arrived at by adjoining to $G_{0}(\bar{A})$ new transformations of the set $A$. The basic problems then are of the following type: Given an extension $G(\bar{A})$ of $G_{0}(\bar{A})$, find invariants of $G(\bar{A})$ which characterize it.

Analogous problems can be formulated in the theory of abstract groups [2]. Now the fundamental structure itself is an abstract group $\bar{A}$. Its automorphism-group $G_{0}(\bar{A})$ may be extended to a group $G(\bar{A})$, by adjoining new transformations of the set $A$. The groupmultiplication of $\bar{A}$, the characterizing invariant of $G_{0}(\bar{A})$, is not invariant under $G(\bar{A})$. The basic problems, stated above for geometry, take the same form in group theory, namely, what are characterizing invariants for $G(\bar{A})$.

A study of this type has already been made in the case $G(\bar{A})$ is taken to be the holomorph $H(\bar{A})$, i.e., the group of transformations obtained by adjoining to $G_{0}(\bar{A})$ the translations of $\bar{A}[4]$. The present paper deals with the case $G(\bar{A})=G_{1}(\bar{A})$, the group consisting of the automorphisms and anti-automorphisms of $\bar{A}$. Characterizing invariants of $G_{1}(\bar{A})$ are investigated.

In a group $\bar{A}$ on a set $A$ one can define two multiplications $p(x, y)$ $=x \cdot y$ and $q(x, y)=y \cdot x$. The automorphism group $G_{0}(\bar{A})$ consists of the automorphisms of the operation $p$, while the anti-automorphisms are those transformations $T$ of the set $A$ which interchange $p$ and $q$. It follows that the set $\{p, q\}$ is invariant under all transformations belonging to the group $G_{1}(\bar{A})$ consisting of the automorphisms and anti-automorphisms of $\bar{A}$. Furthermore, this invariant characterizes $G_{1}(\bar{A})$, i.e., every transformation $T$ of the set $A$ which keeps the set $\{p, q\}$ invariant belongs to $G_{1}(\bar{A})$. However, there are simpler characterizing invariants for $G_{1}(\bar{A})$, namely relations whose arguments

\footnotetext{
Received by the editors September 13, 1955, and, in revised form, December 11,
} 1955. 
range over the set $A$. For example $G_{1}(\bar{A})$ clearly is the group of automorphisms of the 6 -term relation $\beta$ defined as follows:

$$
\beta(x, y, z, u, v, w):(x y=z \wedge u v=w) \vee(y x=z \wedge v u=w) .
$$

Theorem 1 shows that even a 3 -term relation will serve to characterize $G_{1}(\bar{A})$.

THEOREM 1. The automorphisms and anti-automorphisms of a group $\bar{A}$ constitute the group of automorphisms of the relation

$$
\alpha(x, y, z): x y=z \vee y x=z
$$

i.e., the relation $\alpha$ is a characterizing invariant for $G_{1}(\bar{A})$.

The significance of this theorem is that it shows how to replace the rather complex relation $\beta$ by a simpler relation $\alpha$ which is a characterizing invariant for the same group of transformations. It is natural to ask whether this result can be further improved. The relation $\alpha$ is a disjunction of two equations. The question is whether there is a relation expressible in the form of a single equation, which characterizes the group $G_{1}(\bar{A})$ of automorphisms and anti-automorphisms. The answer is negative, it is possible to prove.

THEOREM 2. There is no finite or infinite set of relations expressible as equations between words, which would constitute a system of invariants characterizing the group $G_{1}(\bar{A})$ of automorphisms and antiautomorphisms. I.e., there is a group $\bar{A}_{0}$ and a transformation $T$ on $A_{0}$ such that every equation between words which in every group $\bar{A}$ is invariant under $G_{1}(\bar{A})$ is also invariant in $\bar{A}_{0}$ under $T$, and such that $T$ is not a member of $G_{1}\left(\bar{A}_{0}\right)$.

In algebra one prefers to deal with operations rather than relations. An operation on the set $A$, which characterizes $G_{1}(\bar{A})$, is given in $\S 3$. However, such an operation is neither definable explicitly by a word, nor is it definable implicitly as a solution of an equation of grouptheory. This follows as a corollary to Theorem 2 .

2. Proofs. Theorem 1 states: If $\bar{A}$ is a group and $T$ is a transformation of the set $A$ having the property that for all $x, y \in A, T(x \cdot y)$ is either equal to $T x \cdot T y$ or equal to $T y \cdot T x$, and $T^{-1}(x \cdot y)$ is either equal to $T^{-1} x \cdot T^{-1} y$ or equal to $T^{-1} y \cdot T^{-1} x$, then $T$ must be an automorphism or an anti-automorphism of $\bar{A}$. In this form the theorem was independently obtained by W. R. Scott [8]. As his proof appears in this journal, our proof of Theorem 1 will be omitted.

Let us define a semi-automorphism of a group $\bar{A}$ to be a mapping which preserves $e$ and the functions $S x=x^{-1}$ and $s(x, y)=x y x$. Let 
$G_{1}(\bar{A})$ and $G_{2}(\bar{A})$ denote respectively the group of automorphisms plus anti-automorphisms, and the group of semi-automorphisms. The proof of Theorem 2 now proceeds as follows: first a complete description of all equations invariant under $G_{1}$ in all groups $\bar{A}$ is given. It then can be seen easily that, in the abelian case, all these equations are invariant also under $G_{2}$. The proof is completed by displaying abelian groups $\bar{A}_{0}$ for which $G_{2}\left(\bar{A}_{0}\right)$ is not contained in $G_{1}\left(\bar{A}_{0}\right)$.

An equation of grouptheory will be called reduced if it is either the equation $e=e$ or then is of the type $a_{1} a_{2} \cdots a_{n}=e$, whereby every $a_{i}$ is of the form $x$ or $x^{-1}, x$ being a variable, and none of the pairs $a_{i} a_{i+1}$ and $a_{1} a_{n}$ is of the form $x x^{-1}$ or $x^{-1} x$. Clearly, to every equation $f=g$ one can find a reduced equation $h=e$, such that $(f=g) \leftrightarrow(h=e)$ holds in all groups. It follows that every relation expressible by an equation $f=g$ can also be expressed by a reduced equation $h=e$. In describing the equational invariants of $G_{1}$ and $G_{2}$ it therefore is sufficient to deal with reduced equations only. This procedure will be followed in the sequel. Furthermore, the following notations will be used: Let $x$ be a variable, then $[x]$ stands for $x^{-1}$ and $\left[x^{-1}\right]$ stands for $x$. Let $w$ be a word of grouptheory, i.e., an expression $a_{1} a_{2} \cdots a_{n}$, whereby every $a_{i}$ is of the form $x$ or $x^{-1}$. Then $w^{*}$ stands for the word $a_{n} \cdots a_{2} a_{1}$, and $[w]$ stands for the word $\left[a_{1}\right]\left[a_{2}\right] \cdots\left[a_{n}\right]$. The symbol " $\approx$ " is used to denote syntactic identity of words.

L1: If $g=e$ and $h=e$ are reduced equations such that $(g=e) \leftrightarrow(h=e)$ is true in all groups, then $h$ results by a cyclic permutation of the constituents of either $g$ or $\left[g^{*}\right]$.

To prove this one best uses Gödel's completeness theorem for first order predicate calculus. It says that in $\mathrm{L} 1$ one can replace "true in all groups" by "provable in first-order group theory." Although the validity of the resulting meta-group-theoretic statement is fairly obvious on intuitive grounds, its proof is rather lengthy and therefore it is omitted.

Next we define a reduced equation $g=e$ to be regular 1 in case $g^{*}$ results from $g$ by a cyclic permutation, and to be regular ${ }_{2}$ in case $[g]$ results from $g$ by a cyclic permutation.

L2: If the reduced equation $g=e$ in all groups $\bar{A}$ is invariant under $G_{1}(\bar{A})$, then it is either regular or $_{\text {regular }}$, or $g$ is $e$.

Proof. Suppose $g=e$ is reduced and invariant under $G_{1}$ and $g$ is not $e$. Then $g=e$ is invariant under $S x=x^{-1}$, i.e., $(g=e) \leftrightarrow([g]=e)$ holds in all groups. Therefore by $L 1,[g]$ results from $g$ or $\left[g^{*}\right]$ by cyclic permutation. Consequently $g^{*}$ or $[g]$ results from $g$ by cyclic permutation, i.e., $g$ is regular. Q.E.D.

The next step is to investigate the invariants under $G_{2}$ of regular 
equations. For this purpose the structure of regular equations has to be described. This is done in L4.

L3: Let $g$ be a word of length $n$, and let $P$ be the cyclic permutation of $n$ objects through $m$ places. If $P g \approx g$, then there is a word $w$, such that $g \approx w w \cdots w$ and $m$ is a multiple of the length $l$ of $w$.

Proof. Let $g$ be the word $a_{1} \cdots a_{n}$. The equation

$$
a_{i} \approx a_{i+n}, \quad \text { for all integers } i,
$$

clearly defines a function $i \rightarrow a_{i}$ of the integers into the set $\left\{a_{1}, \cdots, a_{n}\right\}$, which is periodic with period $n$. Because $P g \approx g$, it follows that the function $i \rightarrow a_{i}$ is also periodic with period $m$, i.e.,

$$
a_{i} \approx a_{i+m}, \quad \text { for all integers } i .
$$

Let $l$ be the largest common divisor of $n$ and $m$. Then, $l=p m+q n$ for some integers $p$ and $q$. Therefore, by (1) and (2), the function $i \rightarrow a_{i}$ is also periodic with period $l$, i.e.,

$$
a_{i} \approx a_{i+l}, \quad \text { for all integers } i .
$$

Let $w$ be the word $a_{1} \cdots a_{l}$. Because $l$ divides $n, g$ is of the form ww... Because $l$ divides $m, m$ is a multlple of the length $l$ of $w$. Q.E.D.

L4: If the equation $g=e$ is regular ${ }_{1}$, then the word $g$ must be of the form $g_{1} g_{2}$, whereby both $g_{1}$ and $g_{2}$ are symmetric words, i.e., $g_{1}=g_{1}^{*}$ and $g_{2}=g_{2}^{*}$.

If the equation $g=e$ is $\operatorname{regular}_{2}$, then the word $g$ must be of the form $v[v] v[v] \cdots v[v]$, whereby $v$ is some word.

Proof. Let $g=e$ be regular ${ }_{1}$. Then there is a number $i$ such that a cyclic permutation of $g$ through $i$ places yields $g^{*}$. It may be assumed that $i$ is less or equal to half of the length of $g$, so that $g$ is of the form $a_{1} \cdots a_{j} b_{1} \cdots b_{i}$ whereby $i \leqq j$. The cyclic permutation of $g$ through $i$ places then yields $b_{1} \cdots b_{i} a_{1} \cdots a_{j}$, while $g^{*}$ is the word $b_{i} \cdots b_{1} a_{j} \cdots a_{1}$. Because these two words are identical it follows that $b_{1} \cdots b_{i}$ is identical with $b_{i} \cdots b_{1}$, and $a_{1} \cdots a_{j}$ is identical with $a_{j} \cdots a_{1}$. Therefore $g$ is of the form $g_{1} g_{2}$, whereby both $g_{1}$ and $g_{2}$ are symmetric.

Next let $g=e$ be regular. Then there is a number $i$ such that the cyclic permutation $P$ through $i$ places takes $g$ into $[g]$, i.e., $P g \approx[g]$. It follows that $P P g \approx P[g] \approx[P g] \approx[[g]] \approx g$, i.e., $P P g \approx g$. By L3, there is a word $w$ of length $l$, such that $g$ is of the form $w \cdots w$ and $2 i$ is a multiple of $l$, say $2 i=s \cdot l$. Suppose first that $s$ is even. Then $i$ would be a multiple of $l$, and therefore, $P g$ would be identical to $g$. Because $P g$ is identical with $[g]$, it would follow that $g$ and $[g]$ are 
identical, which is impossible. Consequently $s$ must be odd, and therefore it follows from $2 i=s \cdot l$, that $l$ is even, and $w$ is of the form $w_{1} w_{2}$, whereby both $w_{1}$ and $w_{2}$ are of length $l / 2$. Thus, the situation is as follows:

$$
\begin{aligned}
g & \approx a a, \text { whereby } a \approx w_{1} w_{2} w_{1} w_{2} \cdots w_{1} w_{2}, \\
P g & \approx b b, \text { whereby } b \approx w_{2} w_{1} w_{2} w_{1} \cdots w_{2} w_{1}, \\
\lfloor g] & \approx c c, \text { whereby } c \approx\left[w_{1}\right]\left[w_{2}\right]\left[w_{1}\right]\left[w_{2}\right] \cdots\left[w_{1}\right]\left[w_{2}\right] .
\end{aligned}
$$

Because $P g \approx[g]$ it follows that $w_{2} \approx\left[w_{1}\right]$, and therefore

$$
g \approx w_{1}\left[w_{1}\right] w_{1}\left[w_{1}\right] \cdots w_{1}\left[w_{1}\right] .
$$

L5: If the equation $g=e$ is regular $_{1}$, then in all groups $\bar{A}$ it is invariant under $G_{2}(\bar{A})$.

If the equation $g=e$ is regular $_{2}$, then in all abelian groups $\bar{A}$ it is invariant under $G_{2}(\bar{A})$.

Proof. Suppose $g=e$ is regular ${ }_{1}$. Then by $\mathrm{L} 4, g=e$ must be of the form $g_{1} g_{2}=e$, whereby $g_{1}$ and $g_{2}$ are both symmetric. It is easily seen that every symmetric word is provably equal to an expression composed from $s(x, y)=x y x$ and $S x=x^{-1}$, furthermore, $g_{1} g_{2}=e$ is provably equivalent to $g_{1}=S\left(g_{2}\right)$. It follows that there are expressions $E_{1}$ and $E_{2}$ in $e, S$ and $s$, such that $(g=e) \leftrightarrow\left(E_{1}=E_{2}\right)$ holds in all groups. Because $E_{1}$ and $E_{2}$ are defined from $e, S$ and $s$, the equation $E_{1}=E_{2}$ must be invariant under the automorphism group $G_{2}(\bar{A})$ of $e, S$ and $s$. It follows that $g=e$ is invariant under $G_{2}(\bar{A})$.

Suppose the equation $g=e$ is regular 2 . Then by L3 it must be of the form $v[v] v[v] \cdots v[v]=e$. In every abelian group $\bar{A}$ this equation is identically satisfied, and therefore invariant under $G_{2}(\bar{A})$. Q.E.D.

L6: There are abelian groups $\bar{A}_{0}$ for which $G_{2}\left(\bar{A}_{0}\right)$ is not contained in $G_{1}\left(\bar{A}_{0}\right)$.

Proof. Let $\bar{A}_{0}$ be a Boolean group, i.e., a group which satisfies the equation $x^{2}=e$ identically. In this group $S x=x$ and $s(x, y)=y$. It follows that $G_{2}\left(\bar{A}_{0}\right)$ consists of all transformations of the set $A_{0}$ which keep $e$ fixed. On the other hand, because $\bar{A}_{0}$ is abelian, $G_{1}\left(\bar{A}_{0}\right)$ consist of all automorphisms of $\bar{A}_{0}$. Clearly $G_{2}\left(\bar{A}_{0}\right)$ is not contained in $G_{1}\left(\bar{A}_{0}\right)$, when $A_{0}$ has more than two elements. (For other examples see Dinkines [7].) Q.E.D.

By L2. and L5. it follows that, for abelian groups $\bar{A}$, if an equation is invariant under $G_{1}(\bar{A})$, then it is also invariant under $G_{2}(\bar{A})$. Because of L6, this yields that the equations invariant under $G_{1}(\bar{A})$ cannot characterize $G_{1}(\bar{A})$. This concludes the proof of Theorem 2 . 
3. Remarks. Since by Theorem $1, \alpha$ and $\beta$ have the same group of automorphisms in any $\bar{A}$, they may be said to be equivalent in Klein's sense [1]. This suggests that a stronger sort of equivalence may be established by finding a definition of $\beta$ in terms of $\alpha$. That this is possible will be shown elsewhere by use of the following stronger form of Theorem 1: If two groups $\langle A, \cdot\rangle$ and $\langle A, *\rangle$ have the same $\alpha$, then they must either be identical or anti-groups of each other. From this it also follows that the $\alpha$-theory is an abstraction ([4]; [5]) of group theory, and that every concept of group theory which is invariant under anti-automorphisms is definable in terms of $\alpha$.

The notion of an anti-automorphism applies to any algebraic system $\bar{A}=\langle A, \cdot\rangle$ consisting of a set $A$ and a binary operation $x \cdot y$. While the relation $\beta$ will still be a characterizing invariant for the group $G_{1}(\bar{A})$ consisting of all automorphisms and anti-automorphisms of $\bar{A}$, this will in general not be the case for $\alpha$. However, our proof for Theorem 1 as well as W. R. Scott's makes use of the associative-law and both cancellation-laws only. Therefore, if $\bar{A}$ is a cancellation-semigroup, then $\alpha$ is a characterizing invariant for $G_{1}(\bar{A})$. The following example shows that cancellation-semi-groups still do not exhaust all systems $\langle A, \cdot\rangle$ for which Theorem 1 holds: Let $A$ be any set and let $x \cdot y=x$. Then $\bar{A}=\langle A, \cdot\rangle$ violates one of the cancellation-laws, however, $G_{1}(\bar{A})$ and the group of automorphisms of $\alpha$ are identical, they both consist of all transformations of the set $A$.

In connection with Theorem 2 it should be noted that it is a statement about invariants which are "uniformally" defined for all groups (general invariants in the sense of Baer [2]). In particular groups it may well happen that the anti-automorphisms may be characterized by an equational relation. Thus, as it is shown by F. Dinkines [7], there are many groups in which the semi-automorphisms are exactly the automorphisms and anti-automorphisms. For these groups the equations $x=e, z=x y x$ clearly constitute a system of characterizing invariants for the group of automorphisms and anti-automorphisms.

As a corollary to Theorem 2 it follows that there is no word $w$ in grouptheory, such that in every group $\bar{A}$ the operation $w_{\bar{A}}$ defined by $w$ is a characterizing invariant for $G_{1}(\bar{A})$. However, there are other ways of uniformly defining operations by the use of expressions in grouptheory. For example consider the function $f_{\bar{A}}(a, b, c)$ which takes the value $c$ or $e$ according to whether $\alpha(a, b, c)$ holds or does not hold in $\bar{A}$. One can recover the relation $\alpha(a, b, c)$ from $f, S x=x^{-1}$ and $e$, by defining: $\alpha(a, b, c)$, if and only if, $(c \neq e \wedge f(a, b, c)=c)$ $\vee(c=e \wedge S a=b)$. It follows that $(e, S, f)$ is a system of characterizing invariants for $G_{1}(\bar{A})$. 
Theorem 2 belongs into meta-group theory, i.e., it is a statement about a first order functional calculus $F[e, \cdot,-1]$ with extralogical primitives $e, \cdot$, and ${ }^{-1}$, and extralogical axioms corresponding to conventional group-axioms. The statement may become false if a different formalization of grouptheory is used, for example the rather nonconventional formalization $F[e, \cdot,-1, f]$ with an additional primitive $f$ and an additional axiom, $f(x, y, z)=n \leftrightarrow((x y=z \vee y x=z) \wedge n=z)$ $\vee(x y \neq z \wedge y x \neq z \wedge n=e)$.

\section{BIBLIOGRAPHY}

1. F. Klein, Vergleichende Betrachtungen über neuere geometrische Forschungen, Erlangen (1872), Verlag von Andreas Deichert.

2. R. Baer, Zur Einfïhrung des Scharbegriffs, Crelle's Journal vol. 160 (1929) pp. 199-207.

3. F. I. Mautner, An extension of Klein's Erlangerprogram: Logic as invariant theory, Amer. J. Math. vol. 68 (1946) pp. 345-384.

4. J. R. Büchi and J. B. Wright, The theory of proportionality as an abstraction of group theory, Math. Ann., vol. 130 (1955) pp. 102-108.

5. - Abstraction versus generalization, Proceedings of the International Congress of Mathematics, vol. 2, 1954, p. 398.

6. J. B. Wright, Quasi-projective geometry of two dimensions, Michigan Mathematical Journal, vol. 2 (1953-1954) pp. 115-122.

7. F. Dinkines, Semi-automorphisms of symmetric and alternating groups, Proc. Amer. Math. Soc. vol. 2 (1951) pp. 478-486.

8. W. R. Scott, Half-homomorphisms of groups, Proc. Amer. Math. Soc. vol. 8 (1957) pp. 1141-1144.

UNIVERSITY OF ILLINOIS AND UNIVERSITY OF MICHIGAN 